\title{
Type-specific serologic diagnosis of respiratory syncytial virus infection, based on a synthetic peptide of the attachment protein $\mathrm{G}$
}

\author{
J.P.M. Langedijk ${ }^{\text {a. }{ }^{*} \text {, W.G.J. Middel }}{ }^{\text {a }}$, W.M.M. Schaaper ${ }^{\text {b }}$, R.H. Meloen ${ }^{\text {b }}$, \\ J.A. Kramps ${ }^{a}$, A.H. Brandenburg ${ }^{\text {c }}$, J.T. van Oirschot ${ }^{\text {a }}$ \\ ${ }^{a}$ Department of Botine Virology, Institute for Animal Science and Health (ID-DLO), P.O. Box 65, 8200 AB Lelystad, Netherlands \\ ' Department of Molecular Recognition. Institute for Animal Science and Health (ID-DLO). P.O. Box 65. 8200 AB Lelystad. Netherlands \\ "Department of Virology, Dijk-igt Hospital, Rotterdam, Netherlands
}

Received 29 September 1995: revised 30 November 1995; accepted 5 February 1996

\begin{abstract}
Peptides deduced from the central hydrophobic region (residues 158-189) of the G protein of bovine and ovine respiratory syncytial virus (RSV) and of human RSV subtypes A and B were synthesized. These peptides were used to develop ELISAs to measure specifically antibodies against these types and subtypes of RSV. We have evaluated the bovine RSV-G peptide in both an indirect ELISA and in a blocking ELISA. Specificity and sensitivity, relative to a routine diagnostic ELISA that detects antibodies against the RSV F-protein in bovine sera, were $98 \%$ and $92 \%$ respectively for the indirect peptide-based ELISA, and $98 \%$ and $98 \%$ for the blocking peptide-based ELISA. In paired serum samples, rises in antibody titer were detected more frequently with the indirect peptide-based ELISA than with the routine F-ELISA. Furthermore, the peptide-based G-ELISAs were able to differentiate between antibodies against BRSV and HRSV, and between those against BRSV and ORSV. In addition, the indirect peptide-based ELISA was selective for HRSV subtype A and $B$ antibodies. This study shows that peptides, corresponding to the central hydrophobic region of the attachment protein $\mathrm{G}$ of several RSVs, can be used successfully as antigens in highly specific and sensitive immunoassays.
\end{abstract}

Kejwords: Respiratory syncytial virus; Peptide; Enzyme immunoassay; Subtype

\section{Introduction}

Human and bovine respiratory syncytial virus (RSV) are highly contagious, major pathogens of

Abbreviations: ELISA, enzyme-linked immunosorbent assay; $\mathrm{mAb}$, monoclonal antibody; RSV, respiratory syncytial virus; HRPO, horseradish peroxidase.

* Corresponding author. Tel.: +31-320-238216; Fax: + 31320-238050; e-mail: j.p.m.langedijk@id.dlo.nl. young children and calves that cause respiratory infections each year (Stott and Taylor, 1985). The virus is classified within the Pneumovirus genus of the Paramyxoviridae. Human respiratory syncytial virus (HRSV) is the most important causative agent of bronchiolitis and pneumonia in infants and young children. Approximately 100000 children are hospitalized in the USA each year as a result of RSV infection. Respiratory disease can account for up to $60 \%$ of morbidity and for around $60 \%$ of mortality 
in feedlot cattle (Edwards, 1989; Healy et al., 1993). Bovine respiratory syncytial virus (BRSV) infections are the major cause of respiratory disease in calves resulting in high economic losses. Efficacious vaccines against RSV are not available. Because different antigenic subtypes are described for HRSV (Johnson et al., 1987) and BRSV (Furze et al., 1994), it is important for epidemiological studies and vaccine developments to monitor the prevailing subtypes in a population. Methods are described for the detection of HRSV subtypes using the polymerase chain reaction (Cane and Pringle, 1992). However, the available immunoassays (Welliver, 1988) are based on whole virus or complete proteins that do not discriminate between subtypes of HRSV nor between different RSV types.

RSV has two major surface glycoproteins: the attachment protein $\mathrm{G}$ and the fusion protein $\mathrm{F}$. Because the $G$ protein, in contrast to the $F$ protein, is highly variable between RSV types, a discriminating assay should be based on the $G$ protein. The $G$ protein is unique for RSV, and exhibits a limited homology between HRSV subtypes (53\% amino acid homology) (Johnson et al., 1987), or between HRSV and ungulate RSV (30\% amino acid homology) (Lerch et al., 1990). However, within the subtypes the amino acid homology is much larger: $>80 \%$ within HRSV-A strains (Cane et al., 1991); >90\% within HRSV-B strains (Sullender et al., 1991) and $>90 \%$ within BRSV strains (Mallipeddi and Samal, 1993).

The G protein of RSV (RSV-G) is structurally and functionally distinct from other viral attachment proteins. RSV-G is a type II membrane protein and contains about $60 \%$ carbohydrate by weight. Approximately $20 \%$ of the carbohydrate moiety are N-linked carbohydrates and $80 \%$ are O-linked carbohy- drates. The latter are linked to the very high number of hydroxy amino acids in the protein. The ectodomain of the $\mathrm{G}$ protein can be described as a small central hydrophobic region, devoid of carbohydrates, located between heavily glycosylated mucinlike regions (Langedijk et al., 1996). The C-terminal part of HRSV-G is an important antigenic region (Rueda et al., 1991: Garcia-Barreno et al., 1990; Sullender, 1995) and according to peptide binding studies, a conserved peptide in the central hydrophobic region (residue 174-188) is immunodominant in HRSV-G (Norrby et al., 1987; Akerlind-Stopner et al., 1990) and BRSV-G (Langedijk et al., 1996).

In this study, we demonstrate that the central hydrophobic region of the $G$ protein of RSV is an important antigenic site. Peptides corresponding to the central hydrophobic region of representative RSV types and subtypes have been tested in various immunoassays.

\section{Materials and methods}

\section{I. Peptide synthesis}

Peptides were selected from the central hydrophobic region of RSV-G that is located between the two mucin-like regions. The central hydrophobic regions (residues 158-189) of representatives of all cloned RSV types and subtypes were synthesized: BRSV-G (Lerch et al., 1990), ORSV-G (Mallipeddi and Samal, 1993), HRSV-G type A (Wertz et al., 1985), and HRSV-G type B (Johnson et al., 1987). Additionally, a peptide corresponding to the immunodominant peptide (residues 174-189) of HRSV-G type A was synthesized.

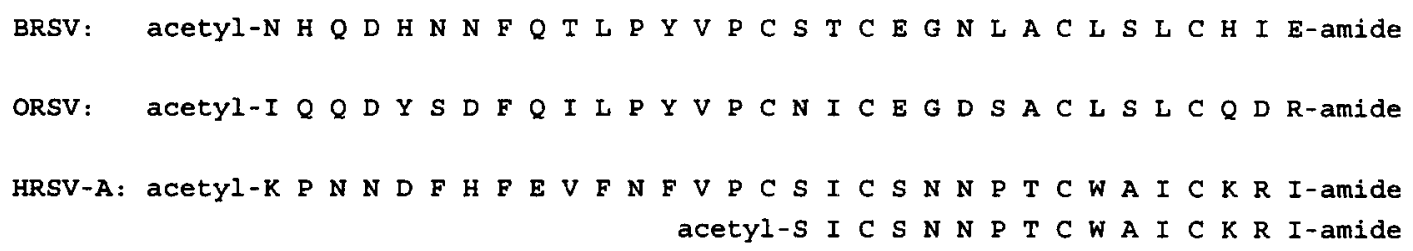


Synthesis of peptides was performed according to standard procedures on an Applied Biosystems 430A synthesizer using Fastmoc chemistry (Fields et al., 1991). Oxidised peptide was obtained from $\beta$ mercaptoethanol reduced peptide that was slowly oxidised by dialysing for 3 days against $1 \%$ $\mathrm{NH}_{4} \mathrm{HCO}_{3}$, which was frequently refreshed. These peptides were used as antigens in enzyme linked immunosorbent assays (ELISA). The purity of the peptides according to HPLC analysis was approximately $40 \%$.

\subsection{Serum samples and monoclonal antibodies}

The following serum samples and monoclonal antibodies were incorporated in the study.

\subsubsection{Cattle}

Negative field serum samples $(n=40)$ were obtained in the summer season from 4-6-month-old calves which had no detectable antibodies against BRSV for at least 1 month (Van der Poel et al., 1993), as determined by the RSV F-ELISA (Westenbrink et al., 1985).

In addition, negative serum samples $(n=12)$ were obtained from specific-pathogen-free (SPF) calves. The calves, obtained by caesarean section, were deprived of colostrum, and reared in isolation.

BRSV-negative serum samples $(n=4)$, containing antibodies directed against either parainfluenza virus type 3 (PI-3), bovine herpes virus 1 (BHV1), bovine viral diarrhea virus (BVDV) or mycoplasma, were also incorporated in this investigation.

Positive field serum samples $(n=100)$, that were positive in the F-ELISA, were obtained from several Dutch farms with a history of BRSV infection (Van der Poel et al., 1993). In the indirect BRSV-G peptide-based ELISA (iG-ELISA), 100 sera were tested and in the blocking BRSV-G peptide-based ELISA (bG-ELISA), 97 of these 100 sera were tested.

Paired serum samples $(n=140)$ from 70 different animals were used to test for increases in antibody titer. Sera were collected with a one month interval in December 1990 and in January 1991 at two different Dutch farms (Van der Poel et al., 1993). Some of these sera $(n=24)$ were used to distinguish between reactivity against the BRSV $-G$ peptide or ORSV-G peptide.
Finally, we tested serum samples $(n=6)$ that reacted non-specifically in the F-ELISA. A serum was considered to bind non-specifically if the absorbance in the noncoated well was higher than the cut-off value.

\subsubsection{Sheep}

Sheep sera $(n=3)$, positive for RSV in the FELISA, were obtained from our internal sheep serum bank.

\subsubsection{Human}

Human sera (panel $1, n=23$ ) positive for RSV in a complement fixation test, were a kind gift of J.C. de Jong of the National Institute of Public Health and Environmental Protection (RIVM) in Bilthoven, the Netherlands. These sera were used in the ELISAs to compare the 16-residue peptide with the 32-residue peptide as antigen.

Paired sera (panel 2, $n=6$ ) of three different children (age 0-6 months) with respiratory tract infection taken in the acute phase and one month later were collected. These patients were confirmed to have RSV infection by direct immunofluorescence on cells from nasopharyngeal washings and virus islolation on Hep2 cells. RSV subtype was identified using RSV subtype specific monoclonal antibodies (92-11C for subtype A and 102-10B for subtype B, Anderson et al., 1985) in immunofluorescence on infected Hep2 cells. These sera were used to distinguish between antibody reactivity against the HRSVA $G$ peptide or HRSV-B G peptide.

\subsubsection{Rabbit}

Rabbits $(n=3)$ were immunized with crude culture supernatant of HRSV-A (strain Long) and HRSV-B (strain 9320) infected cells, respectively. 6 $h$ before the cytopathic effect developed, culture medium was replaced by serum-free medium. Infected Hep2 cells were freeze-thawed after an extensive cytopathic effect had developed. Cell culture was centrifuged at $1600 \times g$ for $10 \mathrm{~min}$ and the supernatant was used for immunization. Rabbits were immunized with $1 \mathrm{ml}$ Freund's complete adjuvant (FCA), mixed 1:1 with $1 \mathrm{ml}$ of supernatant.

\subsubsection{Monoclonal antibodies}

Production of a BRSV-G specific monoclonal antibody (mAb 20) was performed as previously 
described (Wensvoort et al., 1986). BALB/c mice were immunized intraperitoneally with $100 \mu \mathrm{g}$ BRSV (Lelystad strain), grown on embryonic bovine tracheal cells, mixed with FCA. The RSV specificity of $\mathrm{mAb} 20$ was determined using an immunoperoxidase monolayer assay (IPMA) as described (Wensvoort et al., 1986). In this assay Vero cells infected with BRSV (strain Lelystad) were used. Additionally, $\mathrm{mAb} 20$ reacted in the BRSV peptide-based $\mathrm{iG}$ - and bG-ELISA.

MAbs 2G and 19G specific for the $G$ protein of HRSV A, were a kind gift from J.A. Melero, National Centre for Microbiology (Madrid, Spain). MAbs 26 and 30, specific for the G protein of HRSV subtype $B$ and $A$, respectively, were a kind gift from J. Furze and G. Taylor, AFRC Institute for Animal Health, Compton, UK.

\subsection{Respiratory syncytial virus specific F-ELISA}

\subsubsection{Test procedure}

The RSV-specific indirect double antibody sandwich assay, used as a routine diagnostic test in our laboratory, was performed essentially as described previously (Westenbrink et al., 1985), except that mAbs, instead of horse anti-RSV serum, were used as capture antibody. In brief, microtiter plates, coated with two mAbs (no. 88953, ID-DLO, Lelystad) directed against BRSV F were subsequently incubated with bovine RSV antigen, the test serum, rabbit anti-bovine immunoglobulin peroxidase (Dakopatts, P159) and substrate chromogen solution. Before use, and after each incubation step. plates were rinsed six times with deionised water containing $0.05 \%$ Tween 80. Dilutions of test sera and reagents were made in 'high-salt' ELISA buffer $\left(8.1 \mathrm{mM} \mathrm{Na} \mathrm{HPO}_{4}, 2.79\right.$ $\mathrm{mM} \mathrm{KH} \mathrm{PO}_{4}, 0.8 \mathrm{M} \mathrm{NaCl}, 2.68 \mathrm{mM} \mathrm{KCl}, 1 \mathrm{mM}$ EDTA, $0.05 \%$ Tween $80, \mathrm{pH} 7.2$ ) containing $4 \%$ horse serum. BRSV antigen stock solution (no. 88915, ID-DLO, Lelystad) was diluted $1 / 2$ (100 $\mu \mathrm{l} /$ well) and incubated for $2 \mathrm{~h}$ at $37^{\circ} \mathrm{C}$. Test sera were diluted $1 / 80(100 \mu \mathrm{l} /$ well $)$ and incubated for $1 \mathrm{~h}$ at $37^{\circ} \mathrm{C}$. Horseradish peroxidase (HRPO) conjugated rabbit anti-bovine immunoglobulin was diluted $1 / 2000(100 \mu \mathrm{l} /$ well $)$ and incubated for $1 \mathrm{~h}$ at $37^{\circ} \mathrm{C}$. The substrate chromogen solution consisted of $10 \mathrm{mM}$ sodium-phosphate buffer $(\mathrm{pH} \mathrm{6.8)}, 0.1 \mathrm{mM}$ EDTA, $0.1 \% \mathrm{w} / \mathrm{v} 5$-aminosalicylic acid, and freshly added $0.005 \% \mathrm{v} / \mathrm{v} \mathrm{H}_{2} \mathrm{O}_{2}$. Incubation with substrate solution was performed overnight at $4^{\circ} \mathrm{C}$. Colour development was measured at $450 \mathrm{~nm}$ (Titertek Multiscan). When sheep, rabbit or human sera were tested, absorbance values higher than two times the average background value of test sera in control wells without antigen, were considered positive. Although the test has been developed for the detection of antibodies specific for BRSV, antibodies against all other RSV types can be detected with the test due to extensive $F$ protein immune-crossreactivity between RSV types and subtypes (Langedijk, unpublished observations).

\subsection{Indirect G-peptide ELISA (iG-ELISA)}

\subsubsection{Test procedure}

The iG-ELISA was based on the test procedure of the F-ELISA as described above with the following modifications. The antigen was directly coated on to the plate. $150 \mathrm{ng}$ of crude oxidized peptide were coated per well (high binding capacity flat bottom microplate, Greiner) in $100 \mu$ l carbonate buffer $(0.05$ $\mathrm{M}), \mathrm{pH} 9.6,4^{\circ} \mathrm{C}$, overnight. The optimal dilution of the peptide to coat the ELISA plates was chosen in such a manner that a maximum binding was obtained as determined in a checkerboard titration. Test sera, diluted $1 / 5(1 / 10$ in the case of human sera), and conjugate were incubated for $1 \mathrm{~h}$ at $37^{\circ} \mathrm{C}$ in 'low salt' ELISA buffer $\left(8.1 \mathrm{mM} \quad \mathrm{Na}_{2} \mathrm{HPO}_{4}, 2.79 \mathrm{mM}\right.$ $\mathrm{KH}_{2} \mathrm{PO}_{4}, 0.5 \mathrm{M} \mathrm{NaCl}, 2.68 \mathrm{mM} \mathrm{KCl}, \quad \mathrm{mM}$ $\mathrm{Na}_{2}$ EDTA, $0.05 \% \mathrm{v} / \mathrm{v}$ Tween $\left.80, \mathrm{pH} 7.2\right)$ containing $4 \%$ horse serum. Subsequently, the test was performed as described above. The conjugates used in the test were anti-bovine $(1 / 2000)$. anti-sheep (1/1000), and anti-human HRPO (1/1000) (Dakopatts). When sheep or human sera were tested, absorbance values higher than twice the average background value of test sera in control wells without antigen, were considered positive. Only the large bovine serum panels provided the opportunity for an accurate cut-off for positivity/negativity.

\subsection{Blocking G-peptide ELISA (bG-ELISA)}

\subsubsection{Test procedure}

This ELISA for measuring BRSV-specific antibodies is based on blocking of the interaction of a 
BRSV-G specific monoclonal antibody (mAb 20) with the coated peptide by peptide-specific antibodies that may be present in the test sample. ELISA plates were coated with 30 ng crude oxidized peptide per well in $100 \mu \mathrm{l}$ carbonate buffer $\mathrm{pH} 9.0,4^{\circ} \mathrm{C}$, overnight. The optimal dilution of the peptide to coat the ELISA plates was chosen in such a manner that a near maximum binding was obtained as determined in a checkerboard titration and that the sensitivity of the test was maintained at a high level. Before use, and after each incubation step, plates were rinsed six times with deionised water containing $0.05 \%$ Tween 80. Plates were subsequently incubated with test serum diluted 1/2, a HRPO-conjugated monoclonal antibody specific for the G-peptide (bovine RSV$\mathrm{mAb} 20$, ID-DLO, Lelystad) diluted 1/5000, and substrate chromogen solution. Incubation with substrate solution was performed overnight at $4^{\circ} \mathrm{C}$. Test sera and conjugate were incubated for $1 \mathrm{~h}$ at $37^{\circ} \mathrm{C}$ in 'low-salt' ELISA buffer containing $4 \%$ horse serum.

Blocking percentages of each test sample was calculated using the optical density at $450 \mathrm{~nm}$ of 'low salt' ELISA buffer containing $4 \%$ horse serum as reference ( $=0 \%$ blocking) according to the following formula:

$$
\begin{aligned}
& \frac{\mathrm{OD} \text { [ow salt buffer + horse serum }-\mathrm{OD}_{\text {test sample }}}{\mathrm{OD} \text { low salt' buffer + horse serum }_{\text {lo }}} \times 100 \% \\
& =\text { blocking percentage of test sample }
\end{aligned}
$$

\section{Results}

\subsection{Selection of peptide}

The proposed location of the small central hydrophobic region of RSV-G (Fig. 1), the possible autonomous folding of this short sequence, the relatively conserved nature, and the sparse potential glycosylation sites makes a peptide representing this short region a promising candidate for use as an antigen in immunoassays. Besides this structurebased approach, empirical methods have shown that a part of the central hydrophobic region is an important antigenic site. The single linear immunodominant region of BRSV-G as determined by peptide binding studies (Langedijk et al., 1996) (residues

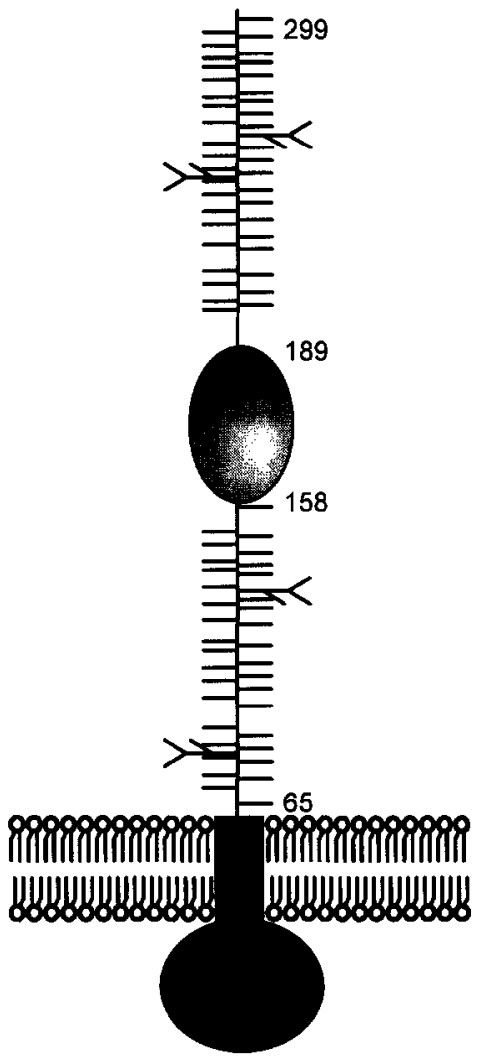

Fig. 1. Schematic structural model of RSV-G protein. The central hydrophobic region (residues 158-189) is shown as a grey ellipse. Mucin-like regions (residues 56-158, 189-299) are shown with potential O-linked glycosylation sites (short horizontal lines) and potential N-linked glycosylation sites (branched lines). Black regions correspond to the transmembrane region and the cytoplasmic region.

174-185) and the immunodominant peptide of HRSV-G described by Norrby et al. (1987) (residues 174-188) correspond to the C-terminal half of the central hydrophobic region of RSV-G (residues 158189). To check whether the empirically determined immunodominant cpitope of HRSV-G type A (contained within the 16-residue peptide 174-189) has the same antigenic characteristics as the predicted antigenic site (the 32-residue peptide corresponding to the central hydrophobic region 158-189), both peptides were tested for their potency to bind specific antibodies in an iG-ELISA with the G-peptide of HRSV subtype $A$ as antigen. Four times more human sera of panel $1(n=23)$ were scored positive 
in the $\mathrm{iG-ELISA}$ based on the 32-residue peptide than in the iG-ELISA based on the 16-residue peptide (data not shown). Although the peptide binding
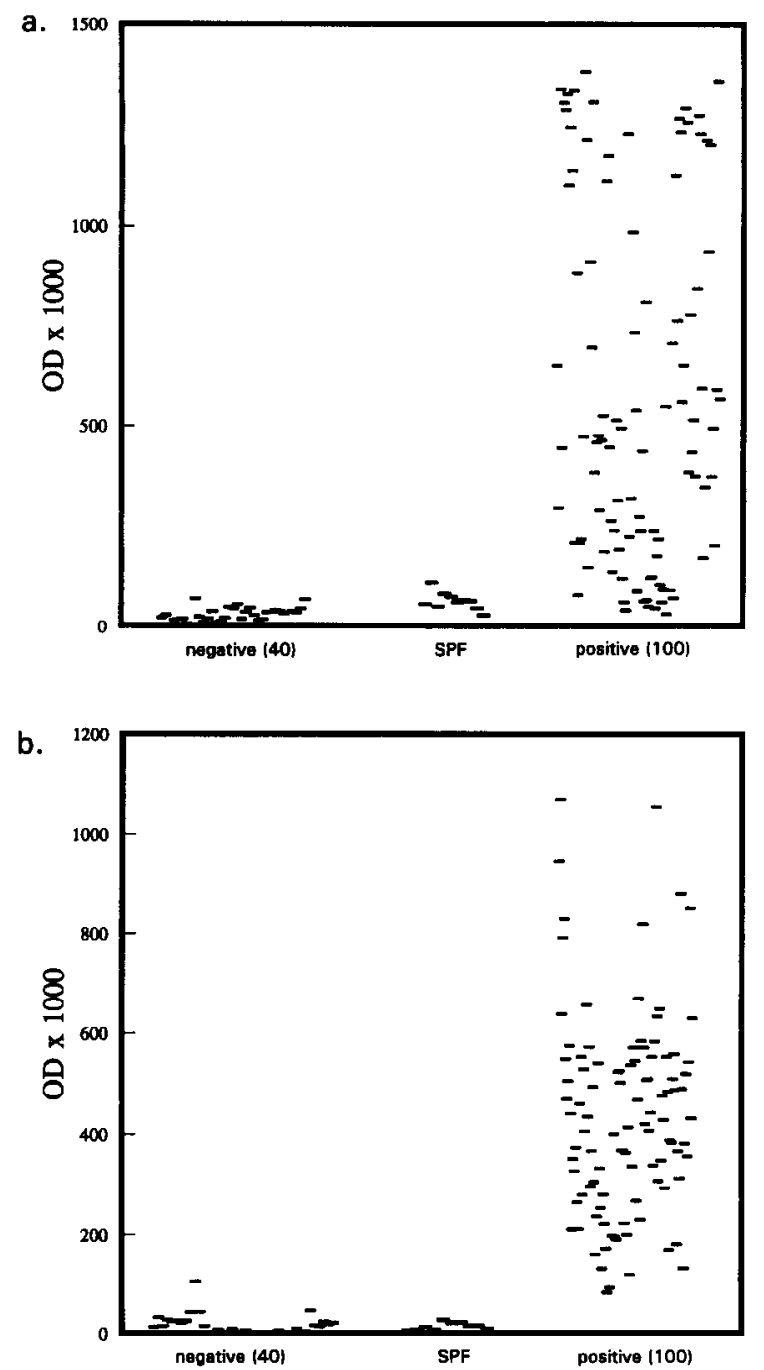

Fig. 2. a: reactivity of bovine sera diluted $1 / 5$ in the indirect BRSV G peptide ELISA as described in the Materials and methods Section 2.4. Negative field serum samples $(n=40)$ were obtained in the summer season from 4-6-month-old calves which had no detectable antibodies against BRSV for at least one month as determined by routine diagnostic F-protein ELISA. Another set of negative serum samples $(n=12)$ was obtained from SPF calves. Positive field serum samples $(n=100)$, that were positive in the F-ELISA, were obtained from several Dutch farms with a history of BRSV infection (Van der Poel et al., 1993). b: reactivity of the same hovine sera, diluted $1 / 80$ in the RRSV F-FI ISA as described in the Materials and methods Section 2.3. studies showed that the immunodominant site is contained in the 16-mer peptide (Langedijk et al., 1996, Norrby et al., 1987), the 32-residue peptide corresponding to the central hydrophobic region of HRSV-G type A as described in this study (Fig. 1), is more reactive with human sera when compared with the 16-residue peptide. Therefore 32-residue peptides, corresponding to the central hydrophobic region of the G-protein of BRSV, ORSV, HRSV-A and HRSV-B were used as antigens in the immunoassays.

\section{2. iG-ELISA}

The reactivity of different panels of bovine sera in the iG-ELISA was compared with the reactivity in the routine diagnostic F-ELISA (Fig. 2). Using the mean OD of all negative sera $(n=40)$ plus twice the standard deviation $(\chi+2 \mathrm{SD}=0.062)$ as cut-off value for negativity, the relative specificity of the iG-ELISA was found to be 0.98 . Using this cut-off value, the sensitivity of the test was determined using 100 positive field serum samples from several Dutch farms and was found to be $0.92(92 / 100)$ when compared to the F-ELISA. Four different sera containing antibodies against other microorganisms (BHV1, BVDV, PI-3, mycoplasma) were all negative in the iG-ELISA (data not shown). Six sera that reacted non-specifically in the routine F-ELIS $\Lambda$ also reacted non-specifically in the iG-ELISA (data not shown).

\section{3. $b G-E L I S A$}

Blocking percentages of the panels of bovine sera were compared in the bG-ELISA (Fig. 3). The sera were the same sera that were tested in the iG-ELISA and F-ELISA (Fig. 2). When the mean blocking percentage of all negative sera $(n=40)(\chi+2$ SD $=42 \%$ ) was used as a cut-off value for negativity, the specificity relative to the F-ELISA was found to be 0.98 . The relative sensitivity of the test as determined using 97 of the 100 positive field serum samples, was found to be $0.98(95 / 97)$. Sera containing antibodies against other microorganisms (BHV-1, BVDV, PI-3, mycoplasma) were all negative in the bG-ELISA (data not shown). The six sera that reacted non-specifically in the routine F-ELISA 


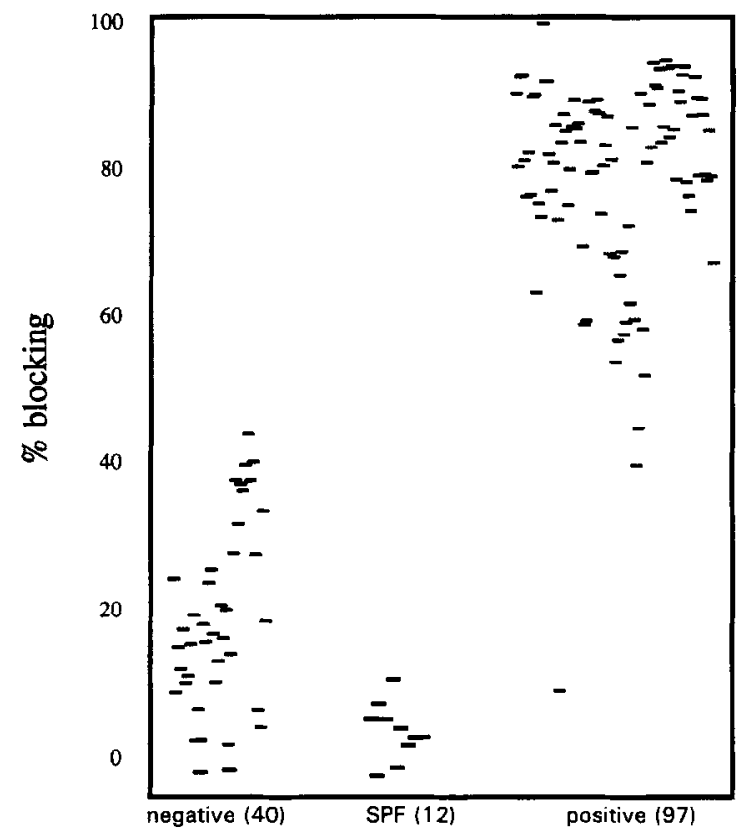

Fig. 3. Blocking percentages of different bovine sera diluted $1 / 2$ in the blocking BRSV $\mathrm{G}$ peptide ELISA as described in the Materials and methods Section 2.5. The same bovine sera were used as in the indirect ELISA shown in Fig. 2. Of the 100 positive sera 97 samples were tested.

and in the iG-ELISA, were tested in the bG-ELISA. Only one of these six sera blocked significantly $(75 \%)$, suggesting that the positive serum contained BRSV specific antibodies.

\subsection{Detection of RSV infection}

An antibody titer rise $(\geq 4 \times)$ in paired sera is normally regarded as being the result of an infection

\section{Table 1}

Frequency of rise in antibody titer $(\geq 4 \times)$, or seroconversion of paired bovine sera in different ELISAs

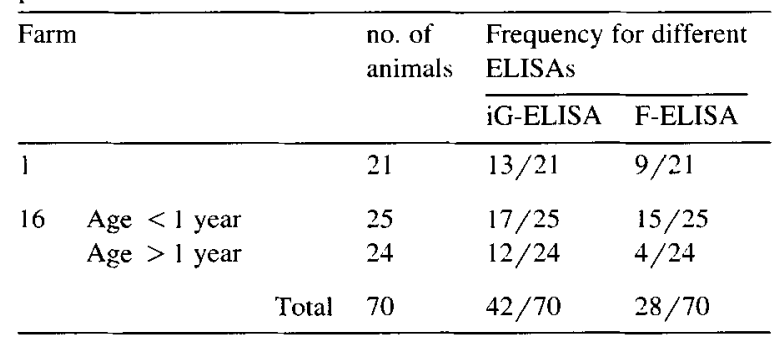

or reinfection. In 70 paired bovine serum samples, seroconversion or at least a fourfold titer rise was detected 42 times in the iG-ELISA and 28 times in the routine F-ELISA (Table 1). Titer rises were detected more frequently in the iG-ELISA than the F-ELISA in animals aged $>1$ year (Table 1 ).

\subsection{Type and subtype specificity of peptide-ELISA}

RSV ELISAs based on whole virus contain antigenic proteins which are very conserved. Therefore, such ELISAs are not type or subtype specific, which means that these assays do not distinguish between antibodies directed against human RSV or ungulate RSV, and certainly not between antibodies directed against HRSV-A and HRSV-B or between BRSV and ORSV specific antibodies. Because RSV-G is highly variable between RSV types and subtypes, we investigated whether peptide-based iG-ELISAs were able to recognize type- or subtype-specific antibodies. ORSV and BRSV are two ungulate RSV types which are genetically equally distant compared to the distance between HRSV subtype A and HRSV subtype $\mathrm{B}$. The genetic distance based on the amino acid sequence of the central hydrophobic region of ORSV and BRSV is comparable to the genetic distance between HRSV-A and HRSV-B. Therefore, the type-specificity of the RSV iG-ELISA can be checked with RSV-positive sera of sheep and cattle, which most likely can only be infected with ORSV and BRSV, respectively. Bovine sera of 24 different

Table 2

Reactivity of bovine and ovine sera in the iG-ELISAs based on the BRSV-G peptide or the ORSV-G peptide "

\begin{tabular}{lcl}
\hline & BRSV G & ORSV G \\
\hline Titer of bovine serum $(n=24)$ & \\
$<5$ & - & 24 \\
5 & 6 & - \\
10 & 2 & - \\
$>40$ & 16 & - \\
Titer of sheep serum $(n=3)$ & \\
$<5$ & 3 & - \\
10 & - & 1 \\
40 & - & 2 \\
\hline
\end{tabular}

${ }^{a}$ Doubling dilutions of sera were prepared, starting with a $1 / 5$ dilution. 
Table 3

Rise in antibody titer. or seroconversion of paired human sera in different ELISAs it

\begin{tabular}{llllll}
\hline Patient & Subtype $^{\mathrm{b}}$ & HRSV-A & iG-ELISA & HRSV-B & iG-ELISA \\
\cline { 3 - 6 } & & T1 & T2 & T1 & T2 \\
\hline 1 & A & $<10$ & 40 & $<10$ & $<10$ \\
2 & A & 10 & 80 & $<10$ & $<10$ \\
3 & B & 10 & $<10$ & $<10$ & 40 \\
\hline
\end{tabular}

"Doubling dilutions of sera were prepared. starting with a $1 / 10$ dilution.

"Subtyping performed as described in the Materials and methods. c $\mathrm{T} 1=$ acute serum, $\mathrm{T} 2=$ convalescent serum.

animals, collected at the same time point, and reacting positively in the routine F-ELISA, were tested for reactivity in the BRSV iG-ELISA and the ORSV iG-ELISA, respectively (Table 2 ). The bovine sera reacted in the BRSV $\mathrm{iG}$-ELISA and not in the ORSV iG-ELISA. In addition, three RSV-positive ovine sera reacted only in the ORSV iG-ELISA and not in the BRSV iG-ELISA (Table 2). Additionally, the reactivity of six paired sera of three children showed that for two children, a specific titer rise was found only in the HRSV-A iG-ELISA and not in the HRSV-B iG-ELISA. For one child a specific titer rise was found for HRSV-B and not for HRSV-A. These specific titer rises corresponded with the infecting virus subtype (Table 3). Furthermore, sera of rabbits immunized with HRSV-A reacted only in the IIRSV-A iG-ELISA, and the serum of the rabbit immunized with HRSV-B reacted only in the HRSV$B$ iG-ELISA. Two HRSV subtype A specific mAbs (2G and $19 \mathrm{G}$ ) reacted only in the HRSV-A iG-ELISA and not in the HRSV-B iG-ELISA. MAb $30 \mathrm{G}$ reacted 60-fold higher in the HRSV-A iG-ELISA compared with the HRSV-B iG-ELISA. HRSV subtype $B$ specific mAb 26 reacted only in the HRSV-B iG-ELISA and not in the HRSV-A iG-ELISA.

\section{Discussion}

The use of peptides as antigens in the serological diagnosis of infections has elicited much interest, because peptides are cheap and easy to produce in a reproducible manner. However, very few peptidebased immunoassays have so far been developed because of lack of sensitivity. Most peptides that have been used in serology represent continuous epitopes. It is impossible to detect antibodies against complex discontinuous epitopes using small linear peptides and it is difficult to predict discontinuous epitopes based on the amino acid sequence of a protein. In addition, the antigenic surface of large globular proteins cannot be mimicked accurately with a small linear peptide. Therefore, the prediction of an independently folding region in a protein that adopts a stable tertiary structure in order to retain its antigenicity, is crucial for the correct design of an antigen. In this respect, our hypothetical model of the ectodomain of RSV-G (Fig. 1) was very helpful for the design of an antigenic peptide (residue 158189). Empirical methods to determine the immunodominant site on BRSV-G and HRSV-G showed that the immunodominant site of the peptide was located within the C-terminal half of this peptide (residue 174-188) (Langedijk et al.. 1996, Norrby et al., 1987). It has been suggested that a 15-residue peptide (residue 174-188) could be used for subtype-specific site-directed serology (Åkerlind-Stopner et al., 1990: Norrby et al.. 1987). However, based on the observation that the complete 32-residue peptide, corresponding to the central hydrophobic region of HRSV-G, reacted better as antigen in an ELISA than the 16-residue peptide (residues 174-189), the 32-residue peptide was used as antigen in the ELISAs.

The BRSV iG-ELISA had a sensitivity relative to the F-ELISA of 0.90 . The low sensitivity compared to the routine F-ELISA may be due to (i) a relative low antigenicity of BRSV-G compared to that of BRSV-F, (ii) low antibody titers of some animals, or (iii) some sera may be directed to another, yet uncharacterized, subtype. In addition to the BRSV iG-ELISA, a more sensitive BRSV bG-ELISA was developed in which antibody detection was based on the capacity to block the reactivity of a $G$ protein specific monoclonal antibody against the peptide. $\Lambda$ sensitivity of 0.98 relative to the routine F-ELISA was obtained, and additionally, this type of ELISA is less prone to false positive results from non-specific sera.

It is more correct to evaluate the performance of a test in the application for which the test is normally used. The RSV ELISAs are normally used to detect infections or reinfections by measuring BRSV- 
specific antibody titer rise in paired sera of animals. The BRSV iG-ELISA was more sensitive in detecting reinfections than the F-ELISA. The difference in frequency of titer increases seemed to be associated with the age of the animal. The iG-ELISA and the routine F-ELISA were equally sensitive for the detection of seroconversion in young calves (age $<1$ year). However, in older cattle (age $>1$ year), four times more antibody titer increases were detected when the iG-ELISA was used. Therefore, the number of reinfections may be markedly underestimated when titer increases are based on the F-ELISA. The different results of both assays may be explained by (i) a faster drop of antibody titers against $G$ compared with that of $F$ after infection, or (ii) by the lower antibody response against $\mathrm{G}$ compared to that against $F$ after the first infection. Consequently, a reinfection may induce a more pronounced increase in antibodies against $G$ than against $F$. Therefore, G-specific ELISAs, including the peptide ELISAs described in this paper, may have an advantage over ELISAs based on the F protein, in that more RSV reinfections are detected.

Another advantage of the peptide-based ELISA is its type specificity and subtype specificity. Bovine and ovine RSV may be considered as two subtypes of ungulate RSV, because the genetic distance between BRSV and ORSV is comparable to the genetic distance between HRSV subtype $\Lambda$ and subtype B (Mallipeddi and Samal, 1993). Bovine sera, positive for RSV in the routine F-ELISA, were positive in the BRSV iG-ELISA and negative in the ORSV iGELISA. Ovine sera, positive for RSV in the routine F-ELISA, were positive in the ORSV iG-ELISA and negative in the BRSV iG-ELISA. Moreover, sera of HRSV-infected children only showed a significant titer rise for antibodies against the HRSV subtype corresponding to the infecting virus subtype. Polyclonal rabbit sera raised against HRSV-A or HRSV-B reacted exclusively with the homologous subtype. Furthermore, four mAbs specific for HRSV-G, also reacted specifically in one of the subtype-specific HRSV iG-ELISAs. An elaborate clinical evaluation is required to determine the most appropriate peptide based ELISA for routine diagnostic application in human and sheep sera.

In conclusion, a short peptide, which corresponds to approximately $0.6 \%$ of the total viral genome, can be used as the antigen in highly sensitive and specific ELISAs. The indirect peptide-based ELISA detected more reinfections in calves than the F-ELISA and can be used for type and subtype specific detection of antibodies against RSV. Therefore, this immunoassay should be valuable for epidemiological studies. Whenever sequence information becomes available about new viral subtypes, a specific peptide ELISA could be easily developed.

\section{Acknowledgements}

The authors wish to thank Drs. J. Furze, J.C de Jong, J.A. Melero, W. van der Poel and G. Taylor for providing us with monoclonal antibodies, human sera and bovine sera, and Franz Daus for technical assistance.

\section{References}

Åkerlind-Stopner, B., Utter, G., Mufson, M.A., Orvell, C., Lerner, R.A. and Norrby, E. (1990) Subgroup-specific antigenic site in the $G$ protein of respiratory syncytial virus forms a disulfidebonded loop. J. Virol. 64, 5143.

Anderson, L.J., Hierholzer, J.C., Tsou, C., Hendry, R.M.. Fernie. B.F., Stone, Y. and McIntosh, K. (1985) Antigenic characterisation of respiratory syncytial virus strains with monoclonal antibodies. J. Infect. Dis. 151, 626.

Cane, P.A. and Pringle, C.R. (1992) Molecular epidemiology of respiratorysyncy tial virus: rapid identification of subgroup $A$ lineages. J. Virol. Methods 40, 297.

Cane, P.A., Matthews, D.A. and Pringle, C.R. (1991) Identification of ariable domains of the attachment $(G)$ protein of subgroup A respiratory syncytial viruses. J. Gen. Virol. 72, 2091.

Edwards, J.A. (1989) The effect of stressors like rumen overload and induced abortion on BRD in feedlot cattle. Agri-Practice 10. 10.

Fields, C.G., Lloyd, D.H., Macdonald, R.L., Otteson, K.M. and Noble, R.L. (1991) HBTU activation for automated Fmoc solid-phase peptide synthesis. Pept. Res. 4, 95.

Furze, J.. Wertz, G.W., Lerch, R. and Taylor, G. (1994) Antigenic heterogeneity of the attachment protein of bovine respiratory syncytial virus. J. Gen. Virol. 75, 363.

Garcia-Barreno B., Portela, A., Delgado, T., Lopez, J.A. and Melero, J.A. (1990) Frame shift mutaions as a novel mechanism for the generation of neutralization resistant mutants of human respiratory syncytial virus. EMBO J. 12, 4181 .

Healy, A.M., Monaghan, M.L., Basset, H.F., Gunn, H.M., Markey, B.K. and Collins, J.D. (1993) Morbidity and mortality in a large Irish feedlot; microbiological and serological findings in cattle with acute respiratory disease. Br. Vet. J. 149, 549. 
Johnson. P.R., Spriggs, M.K., Olmsted, R.A. and Collins, P.L. (1987) The $G$ glycoprotcin of human respiratory syncytial viruses of subgroups A and B: Extensive sequence divergence between antigenically related proteins. Proc. Natl. Acad. Sci. USA 84,5625 .

Langedijk. J.P.M., Schaaper, W.M.M., Meloen, R.H. and Van Oirschot, J.T. (1996) J. Gen. Virol. (in press).

Lerch, R.A., Anderson, K. and Wertz, G.W. (1990) Nucleotide sequence analysis and expression from recombinant vectors demonstrate that the attachment protein $G$ of bovine respiratory syncytial virus is distinct from that of human respiratory syncytial virus. J. Virol. 64, 5559.

Mallipeddi. S.K. and Samal, S.K. (1993) Analysis of the uvine respiratory syncytial virus (RSV) G glycoprotein gene defines a subgroup of ungulate RSV. J. Gen. Virol. 74, 2787.

Norrby, E., Mufson, M.A., Alexander, H., Houghten. R.A. and Lerner, R.A. (1987) Site-directed serology with synthetic peptides representing the large glycoprotein $G$ of respiratory syncytial virus. Proc. Natl. Acad. Sci. USA 84, 6572.

Rueda P., T. Delgado, A. Portela, J.A. Melero and B. Garcia-Barreno. 1991. Premature stop codons in the $G$ glycoprotein of human respiratory syncytial viruses resistant to neutralization by monoclonal antibodies. J. Virol. $65,3374$.

Stotl, E.J. and Taylor, G. (1985) Respiratory syncytial virus: a brief review. Arch. Virol. 84, 1.

Sullender, W.M. (1995) Antigenic analysis of chimeric and trun- cated $G$ proteins of respiratory syncytial virus. Virology 209 , 70.

Sullender, W.M., Mufson, M.A., Anderson, L.J. and Wertz, G.W. (1991) Genetic diversity of the attachment protein of subgroup B respiratory syncytial viruses. J. Virol. 65, 5425.

Van der Poel, W.H.M., Kramps, J.A., Middel, W.G.J., Van Oirschot. J.T. and Van Brand, A. (1993) Dynamics of bovine respiratory syncytial virus infections: a longitudinal epidemiological study in dairy herds. Arch. Virol. 133, 309.

Welliver, R.C. (1988) Detection, pathogenesis and therapy of respiratory syncytial virus infections. Clin. Microbiol. Rev. 1 . 27.

Wensvourt, G., Terpstrat, C., Boonstra, J., Bloemrad, M. and Van Zaane. D. (1986) Production of monoclonal antibodies against swine fever virus and their use in laboratory diagnosis. Vet. Microbiol. 12, 101.

Wertz, G.W., Collins, P.L., Huang, Y., Gruber, C., Levine, S. and Ball, L.A. (1985) Nucleotide sequence of the $G$ protein of human respiratory syncytial virus reveals an unusual type of viral membrane protein. Proc. Natl. Acad. Sci. USA 82, 4075.

Westenbrink. F., Brinkhof, J.M.A., Straver, P.J.. Quak, J. and De Leeuw, P.W. (1985) Comparison of a newly developed enzyme-linked immunosorbent assay with complement fixation and neutralisation tests for serology of bovine respiratory virus infections. Res. Vet. Sci. 38, 334. 